\title{
Contribución al estudio de espesores de soleras de hormigón para cargas de estanterías mediante elementos finitos
}

\section{Study of slab on grade thickness for racking throughout Finite Element Method}

$\underline{\text { C. Ferrer Gisbert }}^{(*)}$, J. J. Ferrán Gozálvez ${ }^{(*)}$, J. B. Torregrosa Soler ${ }^{(*)}$, F. J. Sánchez Romero ${ }^{(*)}$, M. Redón Santafé ${ }^{(*)}$, M. Pérez Sánchez ${ }^{(* *)}$

\section{RESUMEN}

En el presente artículo se realiza un estudio comparativo entre tres procedimientos de cálculo para obtener espesores en soleras de hormigón ligeramente armadas por retracción, con cargas debidas a estanterías. Las fórmulas para cargas aisladas de Westergaard no tienen para este caso una aplicación directa debido a la influencia del resto de soportes y su cercanía. El estudio comparativo se realiza en primer lugar mediante nomogramas de cálculo de la bibliografía; en segundo lugar, la solera se discretiza con elementos finitos y apoya sobre el terreno modelizado con coeficiente de balasto, y en tercer lugar, mediante elementos finitos tridimensionales sólidos que representan la base y la explanada, desarrollándose aspectos novedosos en el tratamiento del conjunto solera-terreno tanto en sus expresiones como en su interacción. Asimismo, se obtienen una serie de gráficas que permiten realizar predimensionados.

Palabras clave: Cargas de estanterías; determinación espesor soleras; MEF.

\section{ABSTRACT}

In the current paper, comparative analyses between three procedures of calculation have been developed, in order to obtain slab on grade thicknesses when the slabs are loaded with racking posts. For this case, the classical expression of Westergaard cannot be applied directly because of the influence of the remainder posts and their proximity. Firstly, the comparative analysis has been done by means of bibliographical design abacus; in the second place, the slab is discretized with finite elements resting above the soil modelled with soil reaction springs; and in third place, a three dimensional solid finite element model represents the base and subgrade set. In the paper some innovative issues regarding the thickness performance and the grade slab interaction are developed. Finally, a series of graphical results are obtained allowing for the pre-design.

Keywords: Racking post loading; slab on grade thickness; FEM.

${ }^{(*)}$ Departamento de Ingeniería Rural y Agroalimentaria. U.D. Construcción. Universidad Politécnica de Valencia (Valencia, España).

${ }^{\left.*^{* *}\right)}$ DIHMA. Universidad Politécnica de Valencia (Valencia, España).

Persona de contacto/Corresponding author: caferrer@agf.upv.es (C. Ferrer Gisbert)

Cómo citar este artículo/Citation: Ferrer Gisbert, C., Ferrán Gozálvez, J. J., Torregrosa Soler, J. B., Sánchez Romero, F. J., Redón Santafé, M., Pérez Sánchez, M. (2016). Contribución al estudio de espesores de soleras de hormigón para cargas de estanterías mediante elementos finitos. Informes de la Construcción, 68(543): e154, doi: http://dx.doi.org/10.3989/ic.15.093.

Licencia / License: Salvo indicación contraria, todos los contenidos de la edición electrónica de Informes de la Construcción se distribuyen bajo una licencia de uso y distribución Creative Commons Attribution License (CC BY) Spain 3.o. 


\section{INTRODUCCIÓN Y ANTECEDENTES}

La determinación de los espesores de solera es un aspecto relevante en todos los almacenes, fábricas y centros logísticos donde las materias primas o los productos terminados ya paletizados se apilan en estanterías. En el campo de la industria agroalimentaria son cada vez más frecuentes, a causa del proceso en sí o por formar parte de los centros de distribución a grandes superficies y a supermercados.

Las dimensiones de las estanterías son variables, según marcas comerciales y procesos, pero lo más habitual es que los largueros permitan el almacenaje de dos palets como máximo (1). El peso de cada palet también depende de la actividad, pero suele estar entre los $7 \mathrm{y}$ los $15 \mathrm{kN}$.

Los autores del presente artículo publicaron otro en esta revista (2), que de alguna manera se quiere extender, realizando la parte del trabajo dedicado a pavimentos rígidos de hormigón para apile en estanterías.

Las fórmulas clásicas de Westergaard (3) y las posteriormente desarrolladas por Pickett y Ray (4) no se pueden utilizar para el cálculo de estanterías al tratarse de expresiones para cargas puntuales aisladas.

El caso de cargas concentradas en soleras de hormigón ha sido abordado por Meyerhof (5) y por Shentu et al. (6) y contrastado por Azzi et al. (7) con posterioridad.

Las expresiones de Meyerhof (5) basadas en la carga última o de colapso constituyen una alternativa a las de Westergaard (3). Son las que recoge, con modificaciones, la actual Technical Report no. 34 de la Concrete Society (8).

En los artículos de Shentu et al. (6) se desarrolla el problema de carga última de una solera para cargas aisladas centradas, por ejemplo, los soportes de un altillo o de una maquinaria, alejados suficientemente entre sí de forma que no existe interacción entre ellos en la transmisión de cargas y sus efectos sobre la solera.

En la citada referencia de Shentu et al. (6) se discretiza el espesor de la solera en elementos finitos apoyándose sobre el terreno modelizado con muelles, y mediante análisis no lineal se observan las líneas de rotura al alcanzarse la carga última. Se deduce también (7) la menor importancia del efecto de punzonamiento frente a los conceptos de radio de rigidez relativa, ya definido desde el punto de vista elástico por Westergaard (3).

Sin embargo, no ha sido resuelto el caso de cargas puntuales de estanterías desde un punto de vista analítico. Debido a la cercanía entre postes o soportes se produce interacción de efectos: tensiones de flexotracción y esfuerzos en la solera, bulbos de tensiones en el terreno, etc.

En los años ochenta se desarrollaron diferentes nomogramas de cálculo apropiados para estanterías (9), (10), (11). Dichos nomogramas se obtuvieron mediante análisis con elementos finitos tipo placa apoyados sobre resortes (terreno). Como los nomogramas tienen sus limitaciones, es decir, sólo ofrecen resultados para determinados coeficientes de balasto, y por tanto, tipo de suelos, se ha querido extender el cálculo comparativo a otros casos, ampliando la gama o serie de combinaciones de suelo estudiadas para el conjunto formado por la base granular y la explanada.

Un procedimiento de cálculo es utilizar líneas de rotura para carga última (6), (7), (8). Otro procedimiento, seguido en el presente artículo, consiste en considerar un diagrama de tensiones elástico que alcanza el límite de flexotracción. De esta forma se consigue evitar que aparezcan grietas en los pavimentos que den lugar a pérdida de condiciones de servicio, funcionalidad, durabilidad, especialmente en la circulación de vehículos estibadores.

El objetivo por tanto es realizar un estudio comparativo entre tres modelos de cálculo, a saber:

- Nomogramas de cálculo del Tecnhnical Report no. 34 (TR34) de la Concrete Society (9). Second Edition.

- Cálculo con MEF utilizando el coeficiente de balasto (pseudo-elástico).

- Cálculo con MEF tridimensional modelizando también el suelo con elementos finitos sólidos tridimensionales.

Para cada uno de ellos se combinan 6 valores de cargas con 5 tipos de explanada y una base granular, proporcionando el espesor por aproximaciones sucesivas y un elevado número de combinaciones de cálculo.

\section{MATERIALES}

\subsection{Geometría del modelo. Patrón de cargas}

La casuística puede ser muy amplia dentro de los distintos sectores industriales, por lo que realizar análisis con todos los tipos de estanterías con sus dimensiones posibles, puede ser largo y tedioso. En el presente artículo se ha ensayado un único modelo, tomado como ejemplo el de una industria cárnica.

El patrón de cálculo (Figura 1) se establece dentro de un recuadro limitado por juntas de retracción, que es lo más desfavorable, sin considerar juntas constructivas con pasadores o similares como se hace en otras referencias (12).

Por simetría, en aras de simplificación y versatilidad de cálculo del modelo, se ha analizado sólo la parte señalada en rojo en el esquema de la Figura 2, derivado de un análisis de sensibilidad previo.

Se añade la simplificación de que la geometría de la placa de anclaje es siempre igual (de 0,2 $\mathrm{m} \times 0,2 \mathrm{~m}$ ), con un área de contacto de $0,04 \mathrm{~m}^{2}$, y las cargas consideradas por soporte de estantería han sido: $50,65,75,85,100,120 \mathrm{kN}$, que cubren la mayor parte de necesidades de almacenaje.

El hecho de que el tamaño de placa de anclaje sea siempre el mismo, permite discretizar los distintos modelos en base a esta unidad (0,2 $\mathrm{m} \times 0,2 \mathrm{~m})$ suponiendo que la base de anclaje es suficientemente rígida. Además, este tamaño permite que para los espesores que se derivan del cálculo en epígrafes posteriores, se verifique la comprobación de punzonamiento según la EHE-08 (apartado 2.2.4.), aunque como se deduce de la experiencia y de la forma de trabajo de las soleras, este tipo de colapso no es habitual en la práctica (7).

Las cargas se introducen como uniformes sobre los elementos finitos en forma de presión, aspecto habitual en el cálculo de pavimentos rígidos, representando el área de contacto. 


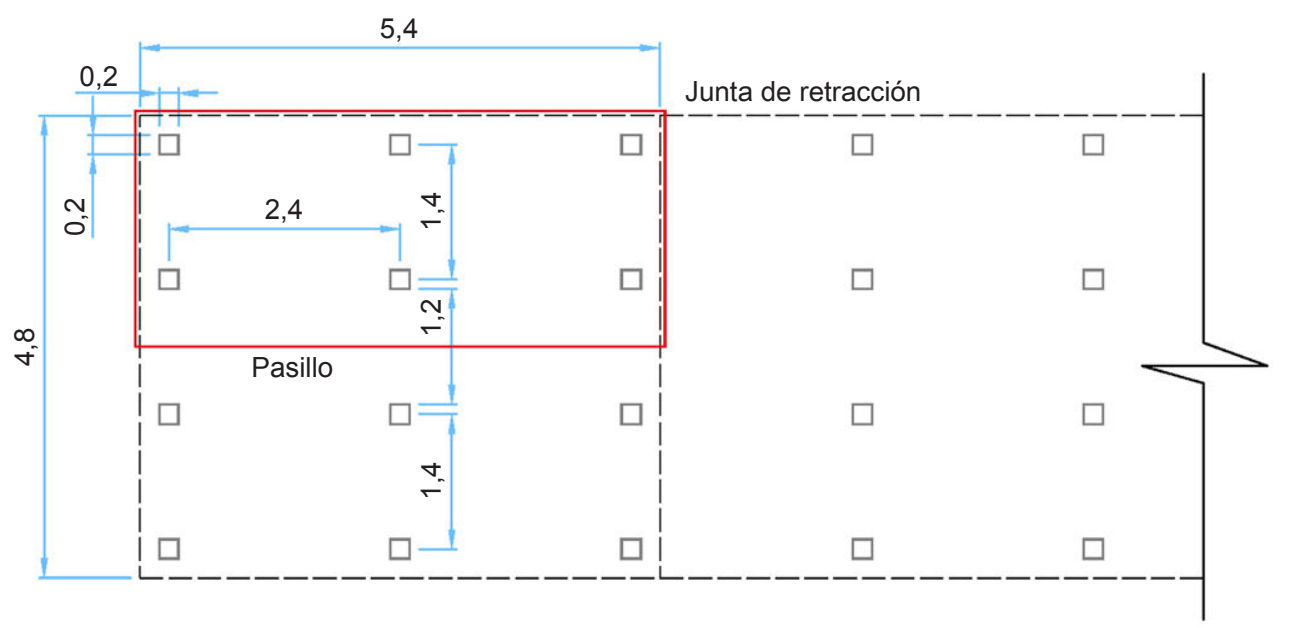

Figura 1. Patrón modelo de cálculo.

\subsection{Características del hormigón}

Las soleras son de hormigón ligeramente armado (HA-25) con un mallazo electrosoldado colocado para disminuir las tensiones de retracción del proceso de curado, o bien con fibras metálicas o una mezcla de ambos elementos.

Desde un punto de vista mecánico el espesor de la solera de hormigón se calcula para que no se vea superada su resistencia máxima a la flexotracción $\left(f_{c t . f l}\right)$, que en el presente artículo se ha seguido la estipulada por la EHE-o8 (13) y por la TR34 (2. edición) (9).

\subsubsection{Resistencia a la flexotracción según EHE-o8}

La resistencia característica a compresión a los 28 días para el hormigón indicado (HA-25) es $f_{c k}=25 \mathrm{~N} / \mathrm{mm}^{2}$.

De acuerdo con los «comentarios» del artículo 39.6, la resistencia media real a los 28 días se puede estimar como $f_{c m}=f_{c k}$ $+8 \mathrm{~N} / \mathrm{mm}^{2}=33 \mathrm{~N} / \mathrm{mm}^{2}$.

Según EHE-o8 (artículo 39) la resistencia media a tracción del hormigón $\left(f_{c t, m}\right)$ es:

$$
f_{c t, m}=0,3 \cdot \sqrt[3]{f_{c k}^{2}}=2,565 \mathrm{~N} / \mathrm{mm}^{2}
$$

Y la resistencia a flexotracción $\left(f_{c t, f}\right)$ :

$$
f_{c t, f l}=\left(1,6-\frac{h}{1.000}\right) \cdot f_{c t, m} \geq f_{c t, m}
$$

Como se puede observar, el valor de la resistencia a flexotracción es linealmente variable con el espesor de la solera $h$ en mm.

A efectos de cálculo se han obtenido los valores comprendidos en el rango que va desde $h=90 \mathrm{~mm}$ hasta $h=400 \mathrm{~mm}$ de milímetro en milímetro, que se ha implementado en una hoja de cálculo para seleccionar debidamente los valores límite.

En los dimensionados se evitará que las tensiones de flexotracción de cálculo para un espesor dado superen estos valores.

Por otra parte, el módulo de deformación secante a los 28 días (artículo 39.6) se obtiene de:

$$
E_{c m}=8.500 \cdot \sqrt[3]{f_{c m}}
$$

Que para HA-25 resulta: $E_{c m}=27.264,04 \mathrm{~N} / \mathrm{mm}^{2}$

\subsubsection{Resistencia a la flexotracción según TR-34 (2. ${ }^{a}$ edición) (9)}

Para todos los casos se proporciona una única expresión con los que se confeccionaron los ábacos de la monografía TR34, como el que se incluye en la Figura 3.

$$
f_{c t, f l}=0,393 \cdot f_{c k}^{\left(\frac{2}{3}\right)}
$$

De donde $f_{\text {ct } f l}=3,360 \mathrm{~N} / \mathrm{mm}^{2}$, más conservadora, por tanto, para espesores de solera inferiores a $290 \mathrm{~mm}$.

\subsubsection{Resistencia a la flexotracción según TR34 (4. ${ }^{\text {a }}$ edición) (8)}

La última edición vigente sigue las especificaciones del Eurocódigo 2 (UNE-EN1992 1-1) (2010): Proyecto de estructuras de hormigón, y, por tanto, coincide con las expresiones de la EHE-o8, ya descritas en 2.2.1.

\subsubsection{Resistencia a punzonamiento (8), (13)}

Con el tamaño de placa elegido $(0,2 \mathrm{~m} \times 0,2 \mathrm{~m})$ y los espesores de cálculo obtenidos se ha hecho una comprobación a punzonamiento, que, aunque no es habitual en pavimentos, es preceptiva según la EHE-o8 (artículo 46) y que también recoge la última edición de la TR-34 (8). Así pues, para dicho tamaño de placa y espesores a partir de $11 \mathrm{~cm}$, se verifica dicha comprobación.

\subsection{Características del suelo}

Se van a analizar cinco tipos de terreno, entendiendo éste como la explanada. Sobre la explanada y por debajo de la solera se extenderá una capa de zahorra artificial seleccionada compactada al 100\% del Próctor Modificado de 0,4 m de espesor en todos los casos como base granular. Es un espesor probado por la experiencia que además de conseguir de «colchón» de reparto de cargas uniforme, evita la ascensión del agua (humedad) por capilaridad. 
La casuística del terreno puede ser muy amplia. A título de ejemplo se ha considerado que la suma de las zahorras artificiales más la explanada es $1,2 \mathrm{~m}$.

Las características de las distintas explanadas y la base granular aplicadas al ejemplo, con carácter general, se recogen en la Tabla 1 (valor resaltado en negrita). Dichos parámetros han sido obtenidos entre horquillas de valores de distintas referencias (10), (11), (14), (15), (16), (17) y cuyos valores también se recogen en la Tabla 1 (máximo y mínimo). Para las propiedades de la zahorra artificial pueden tomarse los valores y características correspondientes al PG3 (18) y la Instrucción 6.1.I-C. (19).

Donde $E$ es el módulo de deformación del terreno y $k_{30}$ el coeficiente de balasto correspondiente a la placa de $30 \mathrm{~cm}$ (17).

Para el segundo método de cálculo se va a utilizar el coeficiente de balasto como elemento soporte de suelo, de acuerdo con la teoría de Winkler (20).

Al tratarse de una sección multicapa se han utilizado las siguientes expresiones [5] y [6] recogidas en las referencias (21), (22) para trabajar con un único coeficiente de balasto medio. Es decir, se han elegido expresiones existentes, cuya novedad reside en su combinación. En la teoría de firmes flexibles se suele recurrir a Burmister (23), procedimiento que resulta más complejo a no ser que se haya implementado en un programa informático (24).

De acuerdo con los trabajos anteriormente citados, el módulo de deformación $E$ medio $\left(E_{s b}\right)$ del conjunto constituido por la base y la explanada se obtiene mediante la expresión [5]:

$$
E_{s b}=\frac{n \cdot(n-1)}{\left[\left(\sum_{i=1}^{n-1} h_{i}\right) \cdot\left(\sum_{i=1}^{n-1} \frac{1}{h_{i} \cdot E_{i}}\right)+\frac{n-1}{E_{n}}\right]}
$$

Donde $n$ es el número de capas, $h_{i}$ el espesor de cada capa y $E_{i}$ el módulo de deformación de cada capa, que varía desde 1 hasta $n$, siendo $n$ la base granular superior.

De acuerdo con Escario et al. (23) se puede obtener el coeficiente de balasto medio kmed a partir de la siguiente expresión aproximada:

$$
\text { kmed } \approx \frac{E_{s b}}{(0,118 \cdot a)}
$$

Siendo $a$ el radio de la placa de 30 pulgadas $=0,762 \mathrm{~m}$, que es la típica que se usa para pavimentos rígidos de hormigón (10), (11). Los valores de $E_{\mathrm{sb}}$ y kmed calculados con las expresiones [5] y [6] para cada uno de los tipos de suelo considerados se recogen en la Tabla 2.

Una relación aproximada de los valores de $k$ viene recogida en la ACI-36o para placa de 12 pulgadas de radio (1 pie, aproximadamente $30 \mathrm{~cm}$ ) y de 30 pulgadas. La correlación entre ambos valores únicamente depende de la superficie de apoyo y es lineal, tal y como recoge Escario et al. (23).

\section{MÉTODOS}

\subsection{Procedimiento de cálculo de la Concrete Society (Technical Report TR34-2. ${ }^{\mathrm{a}}$ edición)}

El Technical Report no. 34 de la Concrete Society, 2. ${ }^{\mathrm{a}}$ edición (1994) (9), recoge en el apartado 5.3.5 una serie de nomogramas para obtener el espesor del pavimento a partir del valor del coeficiente de balasto, que a su vez se puede determinar gráficamente a partir del valor del CBR de las capas subyacentes. Así pues ofrece tres ábacos, uno para $k=15 \mathrm{MN} / \mathrm{m}^{3}$, otro para $k=30 \mathrm{MN} / \mathrm{m}^{3}$ y el último para uno mejorado de $k=55 \mathrm{MN} / \mathrm{m}^{3}$.

Tabla 1. Propiedades físicas y mecánicas de la base granular y explanadas. Valores máximos (máx), mínimos (mín) y valor adoptado

\begin{tabular}{|c|c|c|c|c|c|c|c|c|c|c|}
\hline \multirow{3}{*}{$\begin{array}{l}\text { Identificador } \\
\text { explanada }\end{array}$} & \multirow{3}{*}{ Descripción } & \multirow{3}{*}{ Clasificación } & \multicolumn{2}{|c|}{$\begin{array}{c}\text { Peso específico } \\
\text { aparente } \\
\left(\mathbf{k N} / \mathbf{m}^{3}\right)\end{array}$} & \multicolumn{2}{|c|}{$\mathrm{E}\left(\mathrm{N} / \mathbf{m m}^{2}\right)$} & \multicolumn{2}{|c|}{$\begin{array}{c}\mu \text { (Coeficiente } \\
\text { de Poisson) }\end{array}$} & \multicolumn{2}{|c|}{$k_{30}\left(M N / m^{3}\right)$} \\
\hline & & & Mín & Máx & Mín & Máx & Máx & Mín & Mín & Máx \\
\hline & & & \multicolumn{2}{|c|}{ Valor adopt. } & \multicolumn{2}{|c|}{ Valor adopt. } & \multicolumn{2}{|c|}{ Valor adopt. } & \multicolumn{2}{|c|}{ Valor adopt. } \\
\hline \multirow{2}{*}{ Base } & \multirow{2}{*}{$\begin{array}{l}\text { Zahorra artificial } \\
\text { seleccionada para } \\
\text { base granular }\end{array}$} & \multirow{2}{*}{ GW; A-1-a } & 19 & 23 & 70 & 170 & 0,35 & 0,15 & 138 & $>166$ \\
\hline & & & \multicolumn{2}{|c|}{20} & \multicolumn{2}{|c|}{100} & \multicolumn{2}{|c|}{0,25} & \multicolumn{2}{|c|}{160} \\
\hline \multirow{2}{*}{5} & \multirow{2}{*}{$\begin{array}{l}\text { Explanada suelo duro } \\
\text { (zahorras naturales) }\end{array}$} & \multirow{2}{*}{$\begin{array}{l}\text { A-2 A-3; } \\
\text { GC,SM,SW }\end{array}$} & 19 & 21 & 35 & 55 & 0,40 & 0,2 & 75 & 110 \\
\hline & & & \multicolumn{2}{|c|}{19} & \multicolumn{2}{|c|}{50} & \multicolumn{2}{|c|}{$\mathbf{0 , 3}$} & \multicolumn{2}{|c|}{80} \\
\hline \multirow{2}{*}{4} & \multirow{2}{*}{$\begin{array}{l}\text { Explanada suelo } \\
\text { medio (arenas duras, } \\
\text { arcillas consolidadas) }\end{array}$} & \multirow{2}{*}{$\begin{array}{l}\text { A-2 A-3 A-4; } \\
\text { SM,SC, CL, ML }\end{array}$} & 18 & 19 & 20 & 40 & 0,4 & 0,2 & 30 & 83 \\
\hline & & & \multicolumn{2}{|c|}{18,5} & \multicolumn{2}{|c|}{30} & \multicolumn{2}{|c|}{$\mathbf{0 , 3 5}$} & \multicolumn{2}{|c|}{50} \\
\hline \multirow[b]{2}{*}{3} & \multirow{2}{*}{$\begin{array}{l}\text { Explanada suelo } \\
\text { medio (arenas } \\
\text { blandas, arcillas algo } \\
\text { consolidadas) }\end{array}$} & \multirow{2}{*}{$\begin{array}{l}\text { A-2 A-3 A-4; } \\
\text { SM,SC, CL, ML }\end{array}$} & 18 & 19 & 20 & 30 & 0,4 & 0,2 & 30 & 83 \\
\hline & & & \multicolumn{2}{|c|}{18} & & & & & & \\
\hline 2 & Explanada suelo & $A-5 A-7 \quad \longrightarrow \rightarrow$ & 16 & 19 & 4 & 20 & 0,5 & 0,45 & 14 & 25 \\
\hline 2 & blando & 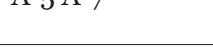 & & & & & & & & \\
\hline 1 & Suelos más blandos & A-6 A-7 & 15 & 18,5 & 4 & 20 & 0,48 & 0,5 & 7 & 21 \\
\hline 1 & 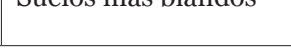 & 20 & & & & & & & & \\
\hline
\end{tabular}
en el ejemplo de cálculo (valor adopt.). 
Tabla 2. Módulo de deformación medio de cada tipo de suelo.

\begin{tabular}{|l|c|c|c|c|c|c|c|c|}
\hline Tipo de suelo & $\begin{array}{c}\text { Identificador } \\
\text { explanada }\end{array}$ & $\boldsymbol{h}_{\mathbf{1}} \mathbf{( m )}$ & $\boldsymbol{h}_{\boldsymbol{n}} \mathbf{( m )}$ & $\begin{array}{c}\boldsymbol{E}_{\mathbf{1}} \\
\left(\mathbf{N} / \mathbf{m m}^{\mathbf{2}}\right)\end{array}$ & $\begin{array}{c}\boldsymbol{E}_{\mathbf{n}} \\
\left(\mathbf{N} / \mathbf{m m}^{\mathbf{2}}\right)\end{array}$ & $\mathbf{n}$ & $\begin{array}{c}\boldsymbol{E}_{\text {sb }} \\
\left(\mathbf{N} / \mathbf{m m}^{\mathbf{2}}\right)\end{array}$ & $\begin{array}{c}\boldsymbol{k m e d} \\
\left(\mathbf{M N} / \mathbf{m}^{\mathbf{3}}\right)\end{array}$ \\
\hline Cohesivo & 1 & 0,8 & 0,4 & 5 & 100 & 2 & 9.804 & 21.807 \\
\hline Cohesivo & 2 & 0,8 & 0,4 & 10 & 100 & 2 & 19.231 & 42.775 \\
\hline Granular & 3 & 0,8 & 0,4 & 20 & 100 & 2 & 37.037 & 82.381 \\
\hline Granular & 4 & 0,8 & 0,4 & 30 & 100 & 2 & 53.571 & 119.159 \\
\hline Granular & 5 & 0,8 & 0,4 & 50 & 100 & 2 & 83.333 & 185.358 \\
\hline
\end{tabular}

La Cement and Concrete Association of Australia obtuvo dichos ábacos en 1985 (25) que después fueron incorporados a la TR34 británica. También ha sido adaptado e incorporado en otras publicaciones como en Jofré et al. (26).

El modo de uso es el siguiente: se entra en abscisas con la carga por soporte (Post load) en $\mathrm{kN}$, se asciende verticalmente hasta intersectar con la tensión de flexotracción máxima del hormigón (Concrete Stress) en MPa; a continuación horizontalmente con el área de contacto en $\mathrm{mm}^{2} \times 10^{3}$, después se traza una línea vertical hasta intersectar con la distancia mayor entre soportes $y$ (larguero) en $\mathrm{mm}$, y luego horizontalmente con la menor $x$ en $\mathrm{mm}$, para ascender verticalmente y en la parte superior obtener el espesor del pavimento en $\mathrm{mm}$.

A título de ejemplo se expone el de $75 \mathrm{kN}$ por soporte (Figura 2).

\subsection{Modelización mediante elementos finitos tipo placa con coeficiente de balasto}

Se ha modelizado y analizado únicamente la zona destacada en rojo en el epígrafe 2, donde el recuadro de solera queda delimitado por juntas de retracción y el modelo analizado se obtiene aplicando el principio de simetría.

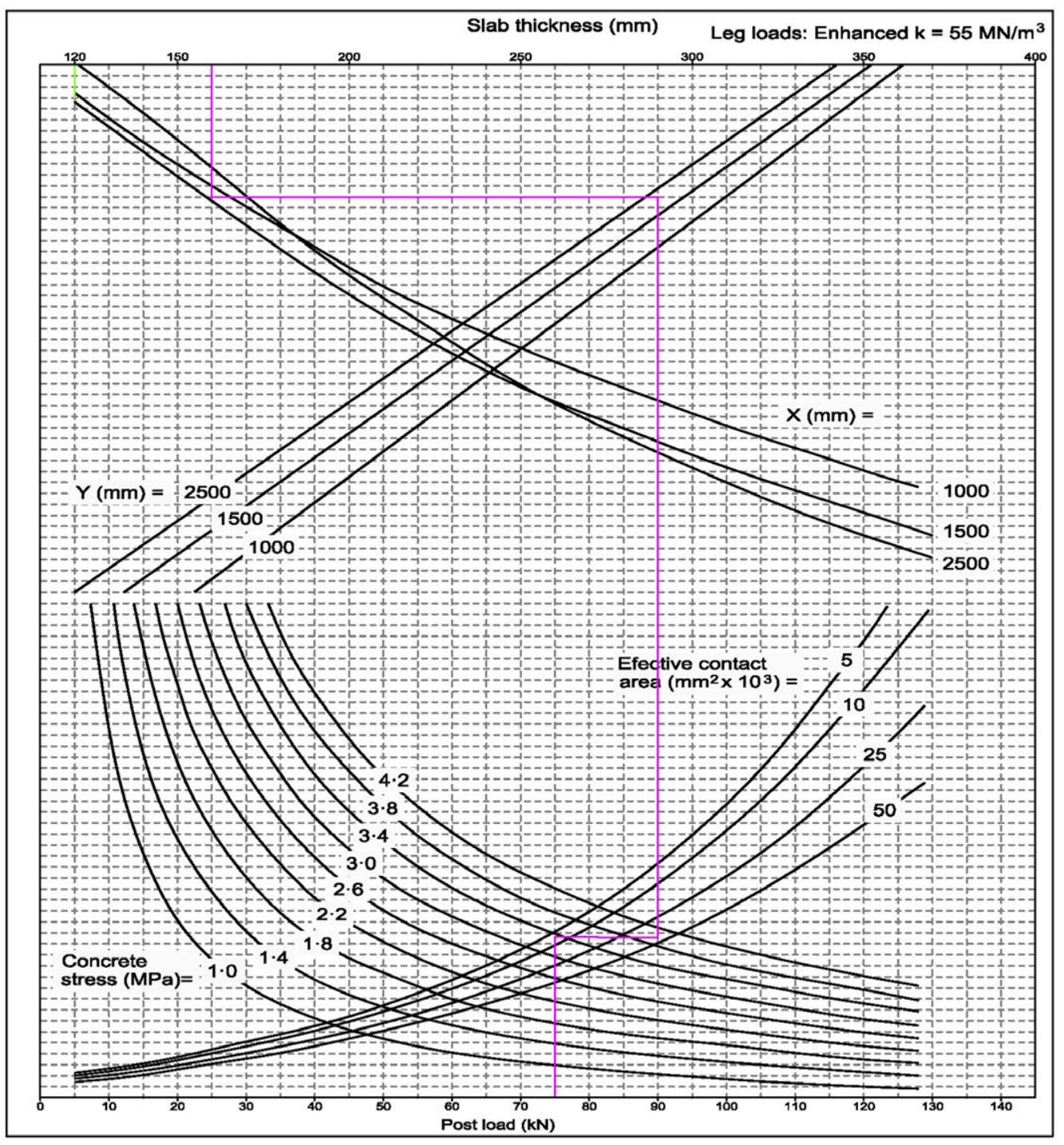

Figura 2. Cálculo con nomogramas TR34 para el ejemplo y una carga de 75kN. 
Se ha utilizado el programa de cálculo por elementos finitos SAP200o (27). La solera se ha discretizado en elementos finitos tipo SHELL de 4 nodos y 6 grados de libertad por nodo, con comportamiento de placa a flexión, apoyado sobre resortes que representan el coeficiente de balasto medio de las cinco situaciones de terreno citadas con anterioridad kmed. También se han repetido los cálculos para poder compararlo con el modelo del TR34-2. ${ }^{a}$ edición, es decir, utilizando un coeficiente de balasto de $k=55 \mathrm{MN} / \mathrm{m}^{3}$.

El cálculo, para cada carga y tipo de suelo, se ha realizado mediante iteraciones sucesivas, no como un trabajo de optimización pero sí como un trabajo de aproximación. Es decir, se han ido modificando los espesores del pavimento para que no se vean superadas las tensiones máximas de flexotracción del hormigón. Se han realizado los cálculos para los seis valores de carga citados.

En la Figura 3 se aprecia el modelo de cálculo.

\subsection{Modelización mediante elementos finitos elásticos tridimensionales}

El método elástico consiste en modelizar el terreno con elementos finitos sólidos tridimensionales.

El modelo se ha realizado con SAP200o utilizando elementos tipo SOLID, hexaédrico, con 8 nodos y 3 grados de libertad por nodo para la base granular y la explanada, y sobre la superficie, la solera se discretiza utilizando elementos tipo SHELL como antes. Para el elemento SOLID es suficiente establecer un comportamiento de modos compatibles (27).

En el apartado de materiales del programa se introducen las características elásticas $(E, \mu, G)$ de cada capa (27).
Se ha utilizado un modelo con 2.808 elementos sólidos y 351 elementos planos, con 10.247 grados de libertad. Teniendo en cuenta las consideraciones del epígrafe 2.1., las condiciones de contorno, por el principio de aislamiento, son: que la base granular y la explanada se encuentran apoyadas horizontalmente en todos sus nodos laterales, permitiéndose el desplazamiento vertical; en la base del modelo los nudos están apoyados verticalmente, y en el eje del pasillo se ha impedido el giro según un eje paralelo al largo (regla de la mano derecha), para simular el momento flector negativo que se obtendría simétricamente en esta zona.

En la Figura 4 derecha se expone un renderizado.

El espesor de zahorra natural se mantiene constante (o,4 m), y se van variando las propiedades de las cinco explanadas. Para cada combinación se han utilizado los seis tipos de carga ya referenciados.

Se establece un sistema híbrido, definiendo de forma independiente la solera (SHELL) y el terreno (SOLID), aunque los nudos de la solera ocupen idénticas posiciones que en la capa superior de los elementos SOLID. En la interfase se relacionan los grados de libertad de ambos tipos de elementos (CONSTRAINTS).

Para cada caso concreto se estudian todas las tensiones en la solera (SHELL) y las tensiones máximas de compresión sobre el terreno (SOLID).

En cuanto a la profundidad del conjunto de elementos finitos, se determinó mediante análisis de sensibilidad, escogiendo un tamaño operativo y funcional de cálculo, y para el cual las tensiones máximas de flexotracción en la solera no variaban y las tensiones en los elementos finitos tipo SOLID apoyados en la base, eran las mismas, para dicha profundidad que para profundidades mayores.
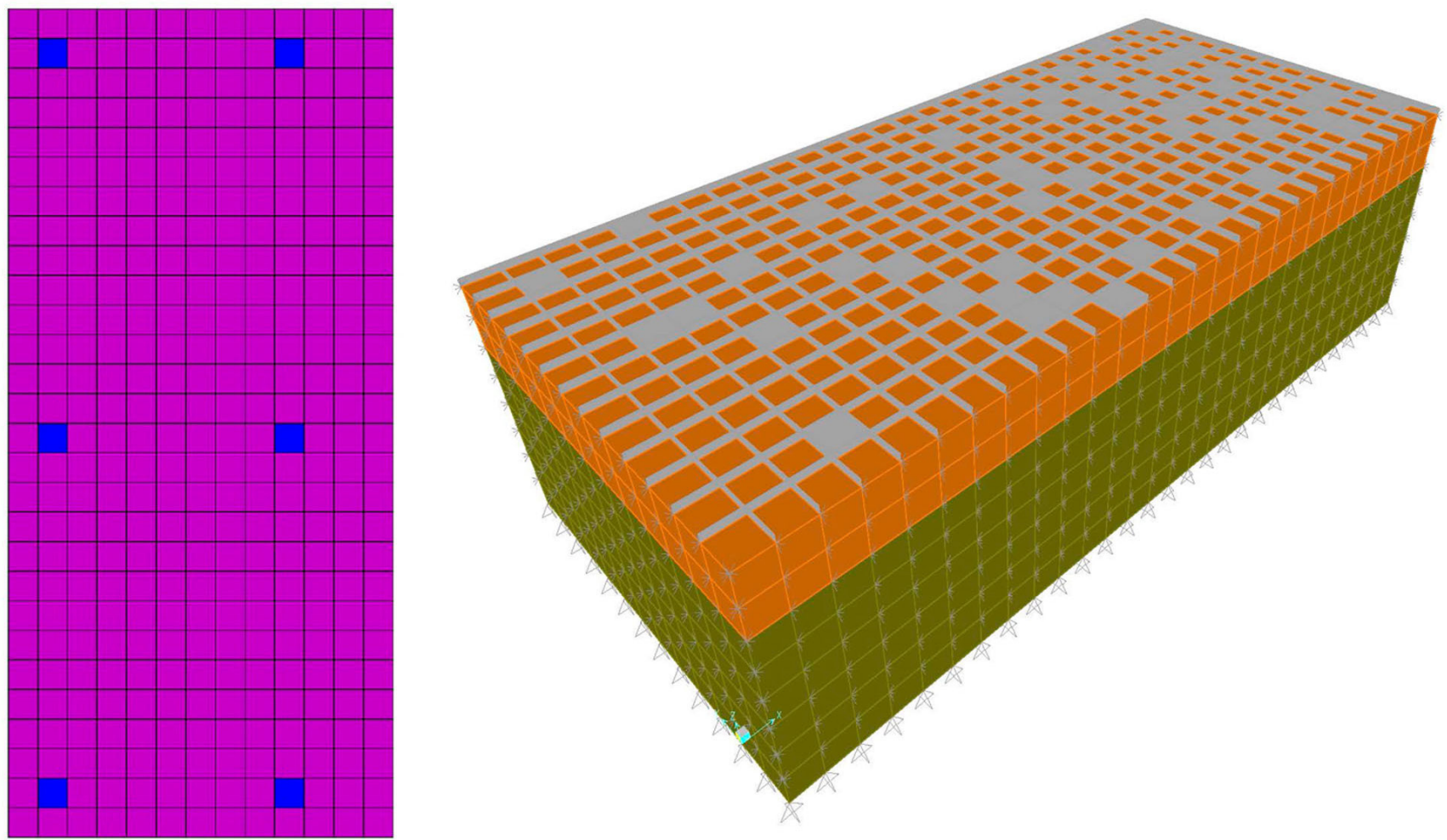

Figura 3. Modelo de cálculo discretizado de la solera con la posición de las cargas introducidas en SAP200o (izquierda). Modelo tridimensional renderizado (derecha). 
Tabla 3. Valores Smax máximos para carga y espesor dados cuya tensión es inferior a la máxima.

\begin{tabular}{|c|c|c|c|c|c|}
\hline $\begin{array}{c}\text { E explanada } \\
(\mathbf{M P a})\end{array}$ & $\begin{array}{c}\text { Kmed conjunto } \\
\left(\mathbf{M N} / \mathbf{m}^{\mathbf{3}}\right)\end{array}$ & $\begin{array}{c}\text { Cargas } \\
(\mathbf{k N})\end{array}$ & $\begin{array}{c}\text { Espesor } \\
\boldsymbol{h}(\mathbf{m m})\end{array}$ & $\begin{array}{c}\text { Smax positiva } \\
\left(\mathbf{M P a} \times \mathbf{1 0}^{-3}\right)\end{array}$ & $\begin{array}{c}\text { Smax negativa } \\
\left(\mathbf{M P a} \times \mathbf{1 0}^{-3}\right)\end{array}$ \\
\hline 300 & 119 & 50 & 111 & $3.783,3$ & $-2.777,4$ \\
\hline 300 & 119 & 65 & 136 & $3.753,5$ & $-2.538,4$ \\
\hline 300 & 119 & 75 & 152 & $3.669,4$ & $-2.390,6$ \\
\hline 300 & 119 & 85 & 165 & $3.640,1$ & $-2.323,6$ \\
\hline 300 & 119 & 100 & 181 & $3.636,5$ & $-2.291,5$ \\
\hline 300 & 119 & 120 & 201 & $3.554,9$ & $-2.243,7$ \\
\hline
\end{tabular}

Por otra parte, si bien es cierto que cuanto más pequeño el elemento finito se obtiene más precisión, tiene inconvenientes en cuanto a la operatividad de cálculo (tiempos, regeneración de imágenes, almacenamiento, etc.). Finalmente se llegó a una solución de compromiso entre la finura del mallado y la operatividad, con la posibilidad de realizar los estudios paramétricos con suficiente número de datos.

En análisis previos se consideraron diferentes tamaños de malla (de $20 \mathrm{~cm} \times 20 \mathrm{~cm}$, tamaño de las bases de anclaje, se pasó a $5 \mathrm{~cm} \times 5 \mathrm{~cm}$ ) y se compararon los resultados. Las variaciones de las tensiones máximas de flexotracción Smax de la solera variaron menos del 2,3\% ante el modelo adoptado con un mayor número de elementos finitos.

Las tensiones que se transmiten son perfectamente asimiladas por la base de zahorra artificial compactada dada su tensión admisible (del orden de o,2 MPa para las situaciones más desfavorables).

El bulbo de presiones que se origina en el terreno (debido al solape) es continuo, es decir, es como si fuera el de un único soporte. Se comprobó que las tensiones son resistidas por las distintas capas.

\section{RESULTADOS}

\subsection{Resultados para procedimiento del método con elementos finitos con coeficiente de balasto}

El programa de cálculo permite la obtención gráfica y numérica de los resultados, para todo tipo de tensiones, aunque las que se han utilizado para el dimensionado han sido Smax (tensiones máximas en las direcciones principales).

En la Tabla 3 se muestran las tensiones máximas obtenidas (Smax) y los espesores de solera necesarios en el caso correspondiente a la explanada $4\left(\mathrm{kmed}=119 \mathrm{MN} / \mathrm{m}^{3}\right.$ y E $=300$ $\mathrm{MPa}$ ) para diferentes valores de carga aplicados.

Para cada caso de terreno la operatividad ha sido la misma.

\subsection{Análisis comparativo entre el método gráfico del TR34 y mediante el MEF sobre coeficiente de balasto}

Se ha repetido la casuística con SAP20oo utilizando un coeficiente de balasto $k=55 \mathrm{MN} / \mathrm{m}^{3}$. De donde comparativamente se obtiene la Figura 4.

Se observa que el método de los elementos finitos empleado resulta menos conservador, excepto para cargas por soporte bajas. No obstante, se aprecia una gran similitud en los resultados.

\subsection{Resultados con procedimiento tridimensional (3D elástico)}

$\mathrm{Al}$ igual que antes se realiza un trabajo de variación del espesor de solera por aproximaciones iterativas con respecto a las tensiones máximas de flexotracción (EHE-o8). Especialmente se ha prestado atención a las tensiones principales máximas positivas (tracción) y máximas negativas (compresión). A título de ejemplo se ofrecen los resultados tabulados correspondientes a la explanada $4 \operatorname{con} E=300$ MPa y diferentes valores de carga (Tabla 4).

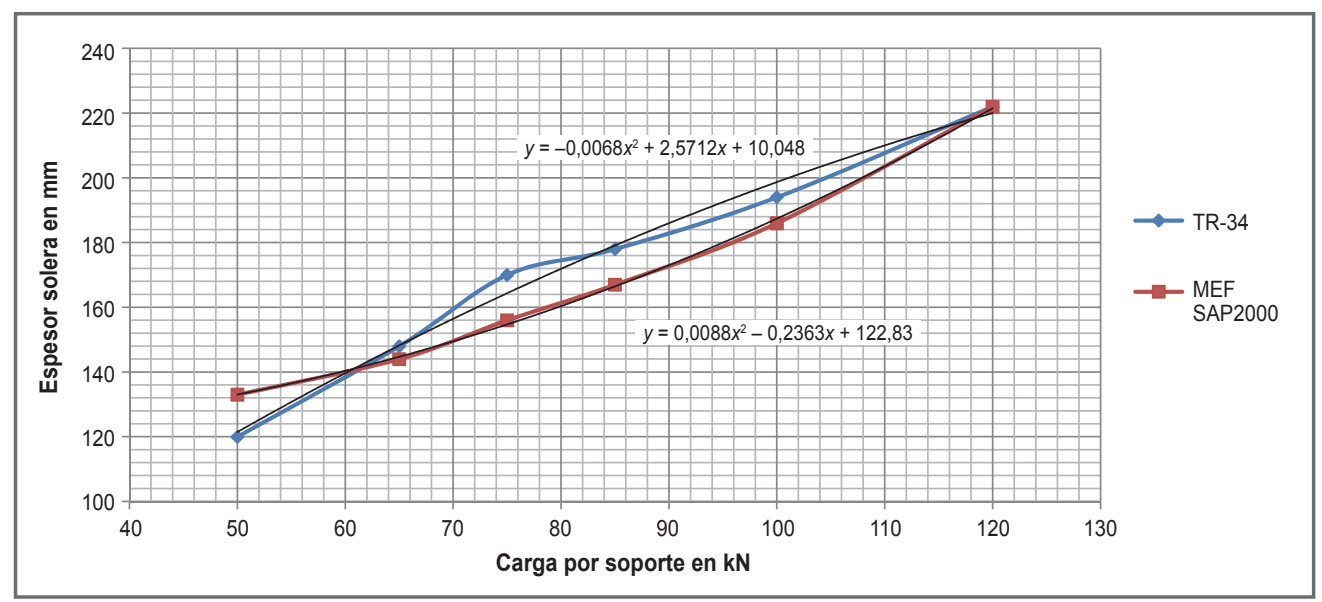

Figura 4. Representación comparativa curvas con SAP200o y nomograma TR34 para $k=55 \mathrm{MN} / \mathrm{m}^{2}$. 
Tabla 4. Tensiones principales máximas en la solera para el caso tridimensional elástico. $E=300 \mathrm{MPa}$.

\begin{tabular}{|c|c|c|c|c|}
\hline $\begin{array}{c}\text { E explanada } \\
(\mathbf{M P a})\end{array}$ & $\begin{array}{c}\text { Cargas } \\
(\mathbf{k N )}\end{array}$ & $\begin{array}{c}\text { Espesor } \\
\boldsymbol{h}(\mathbf{m m})\end{array}$ & $\begin{array}{c}\text { Smax positiva } \\
\left(\mathbf{M P a} \times \mathbf{1 0}^{-3}\right)\end{array}$ & $\begin{array}{c}\text { Smax negativa } \\
\left(\mathbf{M P a} \times \mathbf{~ 1 0}^{-3}\right)\end{array}$ \\
\hline 300 & 50 & 110 & $3.777,3$ & $-2.387,4$ \\
\hline 300 & 65 & 131 & $3.723,9$ & $-2.207,3$ \\
\hline 300 & 75 & 142 & $3.698,4$ & $-2.134,8$ \\
\hline 300 & 85 & 151 & $3.699,6$ & $-2.093,5$ \\
\hline 300 & 100 & 176 & $3.621,7$ & $-1.635,7$ \\
\hline 300 & 120 & 223 & $3.523,4$ & $-1.532,2$ \\
\hline
\end{tabular}

Se observa que en la mayoría de los casos las tensiones de flexotración máximas se producen debido a un momento convexo entre soportes en la dirección mayor o de los largueros entre soportes.

Se ha representado el conjunto de las gráficas en función de carga y espesor (Figura 5).

Se observa como a medida que el módulo de elasticidad de la explanada es mayor el espesor de la solera es menor. También se observa que existe bastante coincidencia a partir de explanadas con $E=200 \mathrm{MPa}$. Mientras que para suelos blan$\operatorname{dos}(E=50$ y $E=100 \mathrm{MPa})$ se observa mayor separación.

El estudio de asientos para el caso más desfavorable, carga de $120 \mathrm{kN}$ y explanada de $E=50 \mathrm{MPa}$, conduce asientos máximos de $4 \mathrm{~mm}$, que también se consideran de poca relevancia.

Como es sabido, para suelos blandos las hipótesis para el estudio de asientos y de tensiones se aleja bastante de la realidad, siendo necesarios métodos edométricos o triaxiales (28), (29).

También se ha representado (Figura 6) la variación del espesor para distintos valores de la explanada y valores de carga. Se observa la misma tendencia de una mayor divergencia para suelos blandos. La figura en sí, cuando las dimensiones $X$ e $Y$ de las estanterías sean similares o no haya grandes diferencias, permite de forma rápida hacer un predimensionado e incluso mediante interpolación.

\subsection{Análisis comparativo entre el método con elementos finitos y coeficiente de balasto y el tridimensional elástico}

Se ha realizado un estudio comparativo entre los resultados obtenidos con el método de elementos finitos con el coeficiente de balasto kmed y el lineal tridimensional elástico, para el conjunto de las cargas y para cada tipo de explanada. Los resultados se muestran gráficamente (Figura 7), donde $k$ representa el método del coeficiente de balasto y $3 \mathrm{D}$ el tridimensional elástico.

Como se observa en las figuras existe cierta similitud entre los métodos. El 3D elástico tiene pendientes más acusadas que el de kmed, especialmente para suelos blandos para los cuales existe una mayor divergencia. Otra observación es que para cargas por soporte altas el método kmed da espesores mayores y con el método elástico del MEF en $3 \mathrm{D}$ es posible afinar mejor el espesor de la solera. En general, cuando las explanadas son más duras, existe una gran convergencia.

\section{CONCLUSIONES}

Los ábacos del TR34 no sirven para cualquier coeficiente de balasto.

En cualquiera de los tres métodos cuanto menor es el módulo de elasticidad o el coeficiente de balasto de la explanada, el espesor es mayor.

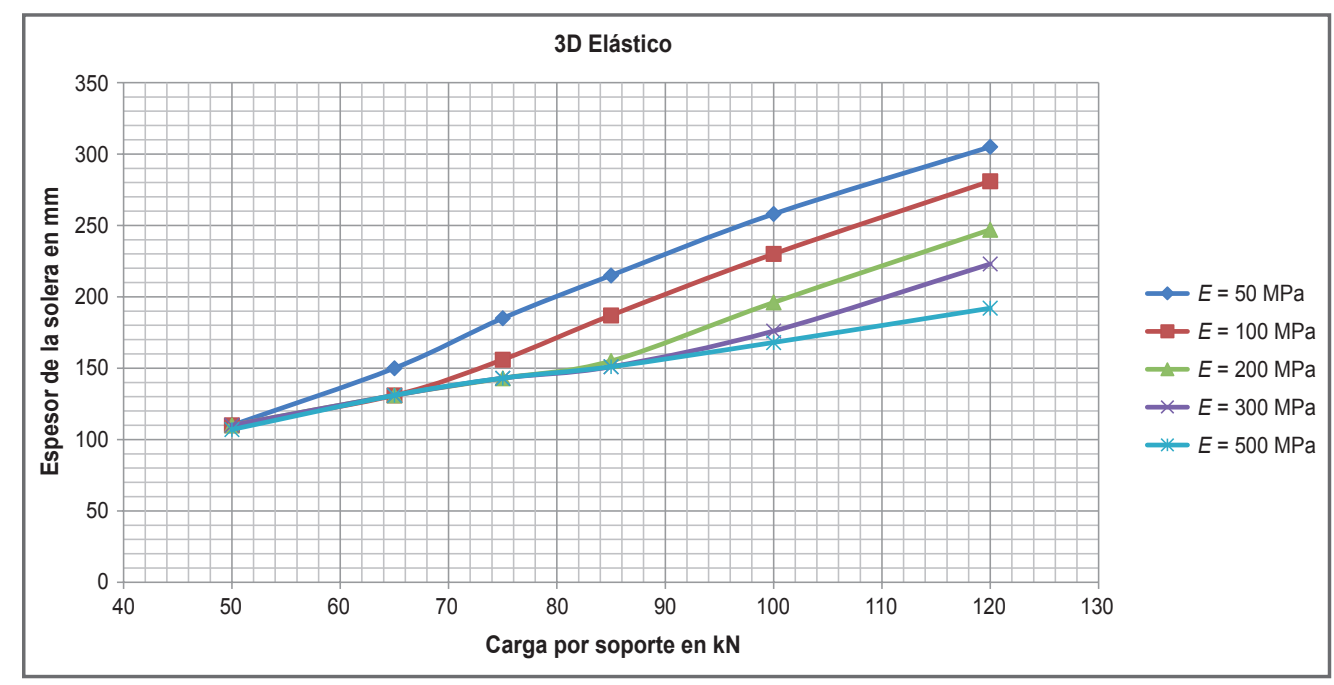

Figura 5. Resultados con método tridimensional elástico en función del tipo de explanada. 


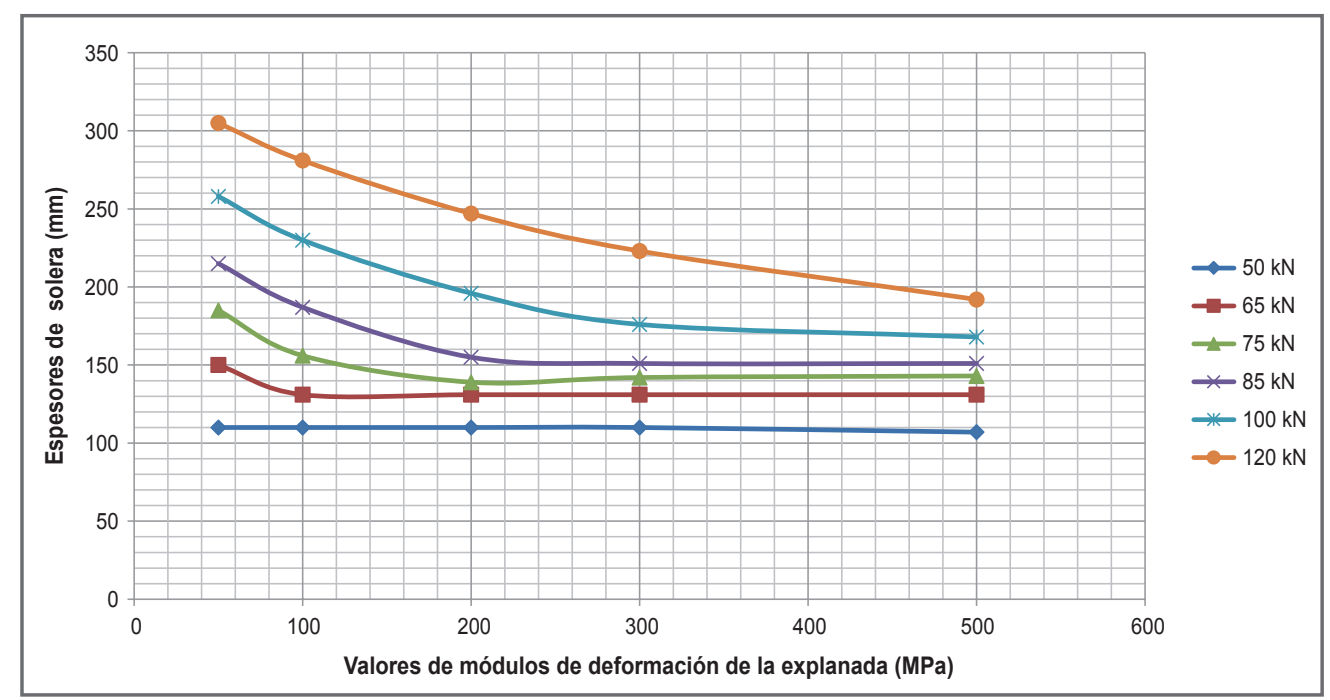

Figura 6. Variación del espesor frente a distintos valores de la explanada y cargas.

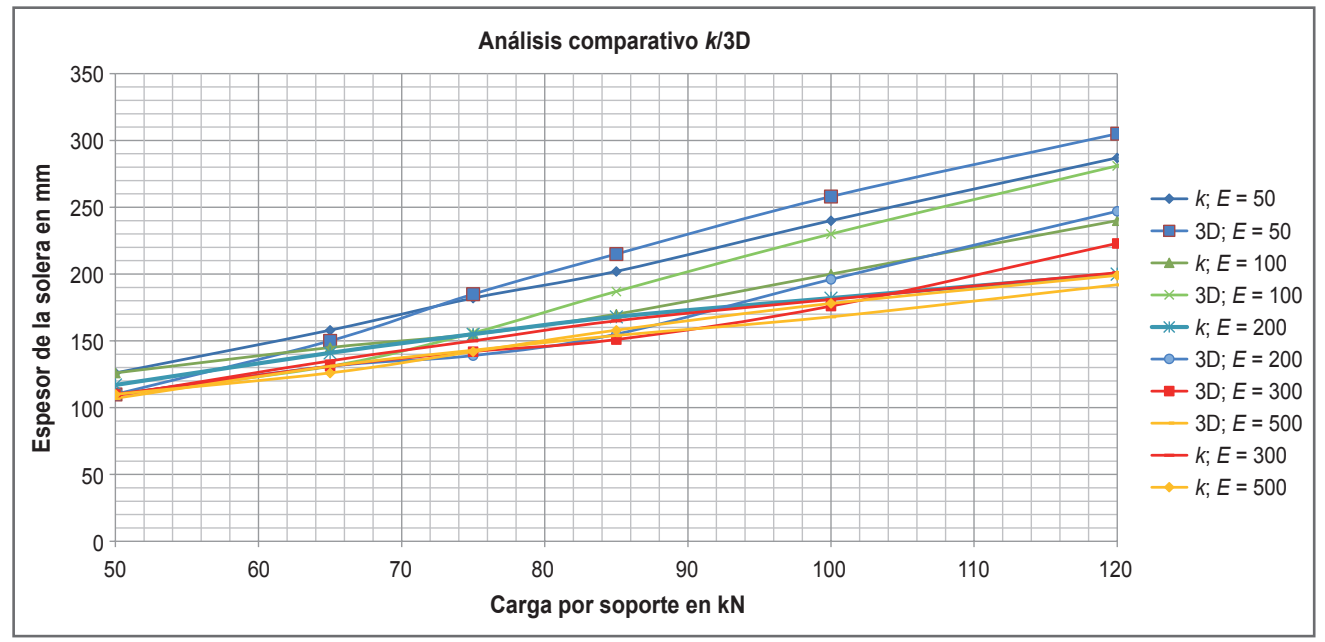

Figura 7. Análisis comparativo.

De los tres métodos los espesores más conservadores se obtienen con los nomogramas del TR34.

El análisis en $3 \mathrm{D}$ con elementos finitos es el que modeliza y representa mejor, y está de acuerdo con la teoría de comportamiento del suelo. En la teoría de Winkler el rozamiento es nulo como es sabido y es un método más aproximado, que destaca por su sencillez.

En los dos modelos numéricos desarrollados aparecen momentos convexos (flexión negativa) en la dirección más gran- de entre largueros, que dan tensiones de flexotracción en la cara superior de la solera, que en muchos de los casos resultan las más desfavorables.

El estudio comparativo entre los dos métodos ofrece gran convergencia para suelos duros, siendo mayores los espesores en el 3D cuando la explanada es blanda.

Por último se ofrece una gráfica obtenida con el método elástico que permite el predimensionado cuando las condiciones geométricas sean parecidas.

\section{REFERENCIAS}

(1) Mecalux, S. A. (2011). Manual técnico del almacenaje. Mecalux, p. 155. Mecalux, S. A.

(2) Ferrer, C., Ferrán, J.J., Ferrer, C.M. (2000). Contribución al estudio, cálculo y diseño de soleras de hormigón en masa para la actividad agroindustrial. Informes de la Construcción, 51(466): 23. CSIC, http://informesdelaconstruccion.revistas.csic.es/index.php/informesdelaconstruccion/article/view/712/797.

(3) Westergaard, H.M. (1926). Stresses in Concrete Pavements Computed by Theoretical Analysis. Public Roads, 7(2): 25.

(4) Pickett, G., Ray, G.K. (1951). Influence Charts for Concrete Pavements. Trans. ASCE, 116: 49.

(5) Meyerhof, G.G. (1962). Load carrying capacity of concrete pavements. Journal of the Soil Mechanics and Foundations Division, Proceedings of the American Society of Civil Engineers. 
(6) Shentu, L., Jiang, D., Hsu, C.T. (1997). Load-carrying capacity for concrete slabs on grade. Journal of Structural Engineering, 123: 95-103.

(7) Azzi, V.D., Laird, R.H. (2008). Load-Carrying Capacity Concrete Slabs-On-Grade Subject to Concentrated Loads. Structure Magazine, April: 18-21.

(8) Technical Report No. 34 (2014) Concrete Industrial Ground Floors - A guide to their Design and Construction, p. 30. Concrete Society. Fourth Edition.

(9) Technical Report No. 34 (1994). Concrete Industrial Ground Floors - A guide to their Design and Construction, p. 69. Concrete Society. Second Edition.

(10) ACI 36oR-92 (Reapproved 1997). Design of Slabs on Grade, p. 43 p.12. American Concrete Institute.

(11) ACI 36oR-10 (2013). Guide to Design of Slabs-on- Ground, p. 59 p.12. American Concrete Institute.

(12) Fwa, T.F. (2006). The Handbook of Highway Engineering, p. 9-53. Taylor \& Francis.

(13) Ministerio de Fomento. (2008). EHE-o8. Instrucción de Hormigón Estructural, p. 167.

(14) Ferrer Gisbert, C. (1998). Contribución al estudio de soleras de hormigón de industrias agroalimentarias mediante la técnica de los elementos finitos. Tesis Doctoral. Universidad Politécnica de Valencia, pp. 164-166.

(15) Rodríguez Ortiz, J.M., Serra Gesta, J., Oteo Mazo, C. (1986). Curso aplicado de cimentaciones, tercera edición, pp. 3234, 162. COAM.

(16) Look, B. (2007). Handbook of Geotechnical Investigation and Design Tables, p. 127. Taylor \& Francis.

(17) Ministerio de Vivienda. (2006). CTE-DB-SE-C. Cimientos, p. 123.

(18) PG3. (2007). Pliego de prescripciones técnicas generales para obras de carreteras y puentes, pp. 229-238. Liteam Ediciones.

(19) Ministerio de Fomento. (2003). Orden FOM/346o/2003, 6.1-IC secciones de firme. Instrucción de carreteras. Boletín Oficial del Estado, n. ${ }^{\circ} 297$.

(20) Winterkorn, H.F., Fang H. (1975). Foundation Engineering Handbook, p. 519. Van Nostrand Reinhold Company.

(21) PCA. (1966). Thickness Design for concrete Pavements. Portland Cement Association.

(22) Papworth, F., Royce, R., Norton, P. Design of steel fibre reinforced concrete slabs on ground and shotcrete linings. Norton Construction Products.

(23) Escario, J.L., Escario, V., Balaguer, E. (1973). Caminos (Tomo II) Firmes de carreteras y aeropuertos, p. 982. ETSI, Caminos, Canales y Puertos, Universidad Politécnica de Madrid.

(24) Everseries $\left.^{(}\right)$User's Guide. (2005). Pavement Analysis Computer Software and Case Studies. Washington State Department of Transportation.

(25) Australia T34. (1985). Concrete Industrial Floor and Pavement Design. Cement and Concrete Association of Australia.

(26) Jofré, C., Vaquero, J.J. (2000). Manual de pavimentos industriales, pp. 60-63. IECA.

(27) Wilson, E.L. (1999). Three Dimensional Static and Dynamic Analysis of Structures. Berkeley, California (USA): Computers \& Structures, Inc.

(28) Ferrer, C.M., Vallés, J.J. (1992). Apuntes de Construcción II. Universidad Politécnica de Valencia.

(29) Mc Carthy D.F. (1993). Essentials of Soil Mechanics and Foundations: Basic Geotechnics, p. 228. Prentice Hall \& Technology. 